\title{
The Computational Complexity of Ball Permutations
}

\author{
Scott Aaronson* \\ Department of Computer Science, UT Austin \\ Austin, Texas, USA \\ aaronson@cs.utexas.edu \\ Greg Kuperberg \\ Department of Mathematics, UC Davis \\ Davis, California, USA \\ greg@math.ucdavis.edu
}

\author{
Adam Bouland ${ }^{\dagger}$ \\ CSAIL, Massachusetts Institute of Technology \\ Cambridge, Massachusetts, USA \\ adam@csail.mit.edu \\ Saeed Mehraban ${ }^{\S}$ \\ CSAIL, Massachusetts Institute of Technology \\ Cambridge, Massachusetts, USA \\ mehraban@mit.edu
}

\begin{abstract}
We define several models of computation based on permuting distinguishable particles (which we call balls) and characterize their computational complexity. In the quantum setting, we use the representation theory of the symmetric group to find variants of this model which are intermediate between BPP and DQC1 (the class of problems solvable with one clean qubit) and between DQC1 and BQP. Furthermore, we consider a restricted version of this model based on an exactly solvable scattering problem of particles moving on a line. Despite the simplicity of this model from the perspective of mathematical physics, we show that if we allow intermediate destructive measurements and specific input states, then the model cannot be efficiently simulated classically up to multiplicative error unless the polynomial hierarchy collapses. Finally, we define a classical version of this model in which one can probabilistically permute balls. We find this yields a complexity class which is intermediate between $L$ and BPP, and that a nondeterministic version of this model is NP-complete.
\end{abstract}

\section{CCS CONCEPTS}

- Theory of computation $\rightarrow$ Quantum complexity theory;

\section{KEYWORDS}

quantum complexity theory, one clean qubit, exchange interaction, intermediate models

\section{ACM Reference format:}

Scott Aaronson, Adam Bouland, Greg Kuperberg, and Saeed Mehraban. 2017. The Computational Complexity of Ball Permutations. In Proceedings of 49th

\footnotetext{
*Supported by a Vannevar Bush Fellowship from the US Department of Defense. ${ }^{\dagger}$ Partially supported by the NSF GRFP under Grant No. 1122374, by a Vannevar Bush Fellowship from the US Department of Defense, and by Scott Aaronson's NSF Waterman award under grant number 1249349.

${ }^{\ddagger}$ Partially supported by NSF grant CCF-1319245.

${ }^{\S}$ Partially supported by a Vannevar Bush Fellowship from the US Department of Defense and by Scott Aaronson's NSF Waterman award under grant number 1249349

Permission to make digital or hard copies of all or part of this work for personal or classroom use is granted without fee provided that copies are not made or distributed for profit or commercial advantage and that copies bear this notice and the full citation on the first page. Copyrights for components of this work owned by others than ACM must be honored. Abstracting with credit is permitted. To copy otherwise, or republish, to post on servers or to redistribute to lists, requires prior specific permission and/or a fee. Request permissions from permissions@acm.org.

STOC'17, Montreal, Canada

(C) 2017 ACM. 978-1-4503-4528-6/17/06 ..\$15.00

DOI: $10.1145 / 3055399.3055453$
}

Annual ACM SIGACT Symposium on the Theory of Computing, Montreal, Canada, June 2017 (STOC'17), 11 pages.

DOI: $10.1145 / 3055399.3055453$

\section{INTRODUCTION}

The standard model of quantum computing is defined using quantum circuits acting on qubits. The computational power of this model is captured by the complexity class BQP, which resides somewhere between the complexity classes BPP and PP [20]. However, physical systems can exist in Hilbert spaces which are not described by tensor products of qubits. For example, systems of noninteracting fermions, noninteracting bosons, or anyons in a $2+1$ dimensional quantum field theory all live in Hilbert spaces with different mathematical descriptions.

A natural problem is to explore the computational complexity of these alternative physical systems. There are several reasons to study this problem. First, many of these alternative models of quantum computing seem to be intermediate in power between classical and quantum computing [2, 14, 25, 35]. Therefore it is interesting to study their power from a purely complexity-theoretic standpoint, as they help delineate the boundary between classical and quantum computation. Second, these models sometimes have special properties from the perspective of mathematical physics. For instance, they might be "solvable" or "integrable" systems, which are regarded as simple to mathematical physicists. It is interesting to compare notions of simplicity in mathematical physics (solvability and integrability) to the notion of simplicity in computational complexity (efficient classical simulability).

Motivated by the above, in this work we consider an alternative model of quantum computing based on permuting quantum balls, and study its computational complexity. More specifically, we consider a system of $n$ distinguishable particles ("balls") on a line. The computational basis of this Hilbert space consists of all permutations of the particles. We denote this Hilbert space by $\mathbb{C} S_{n}$. The quantum operations in this model act on two balls at a time, and map the state $|x, y\rangle$ to the state $|x, y\rangle \rightarrow c|x, y\rangle+i s|y, x\rangle$, where $x$ and $y$ distinct integers from 1 to $n$, and $c$ and $s$ are real numbers with $c^{2}+s^{2}=1$. For example, if one has the state $|123\rangle$, and applies the above operation to the first two particles, the resulting state would be $c|123\rangle+i s|213\rangle$. This is a quantum analog of exchanging the particles in that location with probability $s^{2}$; hence we call this 
the "partial swap" gate ${ }^{1}$. Physically, this "ball-permuting" model captures the scattering problem of distinguishable particles on a line with short-range interactions ${ }^{2}$.

We study the computational complexity of several models based on the above formalism. First, we consider a model in which one starts in the state $|123 \ldots n\rangle$, and then applies polynomially many partial swap gates. We show that one can approximate amplitudes in this model, within 1 /poly additive error, within the one-cleanqubit model of Knill and LaFlamme, also known as the complexity class DQC1 [29]. This class captures the power of quantum computers in which all input qubits are in the maximally mixed state (i.e. a uniformly random basis state unknown to the experimenter) except one, which is in a pure initial state. This model is widely believed to be substantially weaker than BQP; in fact it is open whether or not DQC1 is even capable of universal classical computation. Therefore, the power of this model seems to be substantially weaker than BQP. Our result shows that exchange based quantum computing with distinguishable particles starting in the state $|12 \ldots n\rangle$ yields a weak computational model. This in turn suggests that indistinguishability is a crucial computational resource for exchange based computations.

Next, we consider the computational power of this model when we begin with arbitrary initial states (i.e. states more complicated than $|1,2, \ldots, n\rangle)$. First, we show that if the initial state is selected according to certain irreducible invariant subspaces, then this model can efficiently simulate BQP, using an encoded universality. We also mention an explicit construction based on the result that the exchange interaction on qubits is encoded-universal $[18,19]$. Therefore allowing arbitrary initial states substantially boosts the power of this model.

Furthermore, we obtain a partial classification of the computational complexity of this model on different input states. In order to achieve this goal, we use the representation theory of the symmetric group. In particular, we use the Young-Yamanouchi orthonormal basis [24] to describe subspaces of our Hilbert space that are invariant and irreducible under the action of ball permuting gates. Therefore to understand the complexity of our model starting from an arbitrary input state, one merely needs to analyze its components in the Young-Yamanouchi basis using representation theory. We make progress towards this goal in Section 3.2.

One interesting finding of this classification is the discovery of a natural model of quantum computing which seems to be intermediate between DQC1 and BQP. In this model, we initially start with the input state

$$
\frac{1}{\sqrt{2^{n}}} \sum_{x \in\{0,1\}^{n}}|x\rangle|x\rangle,
$$

consisting of $n$ active qubits maximally entangled to $n$ inert qubits. We imagine we can apply an arbitrary quantum circuit to the active qubits only (i.e. the left half of the state only), and then we measure

\footnotetext{
${ }^{1}$ Note Jordan [25] also uses permutations to obtain an intermediate model, but the operators he considers are different from partial swaps as they do not create superpositions in the particle location basis. Thus they are more analogous to "total swaps".

${ }^{2}$ In the interactions we consider, contact between particles is penalized with a delta function. In this sense the interactions are "hard". However, in the physics literature these are referred to as "soft short-range" interactions since they allow permutation of particles, whereas "hard" interactions forbid the permutation of particles.
}

both active and inert qubits together in the computational basis. If one were to only examine the computation on the left half of the state only ${ }^{3}$, this model would be equivalent to DQC1 - because the left half of the state considered in isolation is maximally mixed. However, in this new model one additionally gets to observe the right half of the state. In other words, although the qubits are initially in an (unknown) uniformly random basis state at the start of the computation, one gets to learn what basis state they started in, but only at the end of the computation. We find that this model arises naturally in ball-permuting circuits starting with input states of specific "reducible subspaces". This model may be of independent interest.

Third, motivated by physical considerations, we consider a restricted version of this model in which the exchange operators are required to satisfy the (parameter dependent) Yang-Baxter equation. The Yang-Baxter equation arises numerous places in mathematical physics, including two-dimensional quantum field theory, statistical mechanics, and topological quantum field theory. The equation describes sets of operators which form representations of the braid group. In our setup the Yang-Baxter equation arises naturally from quantizing a system of $n$ distinguishable particles with their own momenta and short-range [43] interactions ${ }^{4}$. Less formally, this arises if one imagines that the balls have their own velocities, and upon colliding, either exchange velocities (as in classical physics) or else pass through each other, with quantum amplitudes $c$ and is, respectively. In this case one cannot exchange arbitrary particles due to the velocity constraints. This specifically means that the $X$ gate corresponding to the collision of a particle with momentum $p_{1}$ with a particle with momentum $p_{2}$ should have the parameter

$$
c=\frac{1}{\sqrt{1+\Delta\left(p_{2}-p_{1}\right)^{2}}} .
$$

Where $\Delta$ is a constant related to the strength of interactions between the particles.

We show that if intermediate measurements are added to this model, then one cannot sample from the same probability distribution (up to multiplicative error) efficiently with a classical computer unless the polynomial hierarchy collapses to the third level. The proof uses a postselection [1] argument; we show that postselecting on possibly exponentially-unlikely measurement outcomes allows the model to efficiently solve any problem in the complexity class PostBQP. Then using the same line of reasoning as in AaronsonArkhipov [2] and Bremner-Jozsa-Shepherd [14], one can infer that the existence of an efficient procedure to sample from the distribution of outcomes in the proposed model within multiplicative error implies the collapse of polynomial hierarchy to the third level.

This result might be somewhat surprising to mathematical physicists because systems obeying the Yang-Baxter equation in $1+1$ dimensions are considered simple because they are "Bethe ansatz solvable". "Bethe ansatz solvable" models are restricted subclasses of "integrable" models, which themselves are restricted subclasses

\footnotetext{
${ }^{3}$ As well as the first qubit of the right half of the state

${ }^{4}$ Specific to the integrable theories we consider in $1+1$ dimensions, the Yang-Baxter equation is a consistency equation which implies that the unitary evolution depends only on the initial momenta of the particles. In the language of mathematical physics this last condition is also known as Bethe Ansatz solvability.
} 
of "exactly solvable" models ${ }^{5}$. Exactly solvable systems have the property that amplitudes can be computed in all parameter regimes of the system. In contrast, for many physical systems e.g. quantum field theories, sometimes one can only compute amplitudes in a restricted regime of parameters known as "weakly coupled" or "strongly coupled" [36]. Integrable systems have the further property that the number of conserved quantities ${ }^{6}$ exceeds the number of degrees of freedom. So by specifying these conserved quantities, one has fully specified the scattering amplitudes i.e. the unitary matrix describing the evolution of the system; there is no need e.g. to solve a differential equation such as the Schrödinger equation, or compute a sum of Feynman diagrams. Bethe ansatz solvable systems have the further property that the eigenstates of the Hamiltonian take a particular form (known as the Bethe "ansatz" or "guess"). The Bethe ansatz manifestly obeys the Yang-Baxter equation. Furthermore, the Bethe ansatz implies that the scattering amplitudes can be fully specified with linearly many real parameters. In our Yang-Baxter ball-permuting model, these real parameters are the initial particle velocities. As a result, the dimensionality of the set of unitary matrices ${ }^{7}$ allowed in this model grows only linearly in the number of particles, while the dimensionality of the space of all unitary evolutions grows exponentially. Intuitively, it seems difficult to "program" such models to do any interesting computations, since there are few parameters in the system. Our result therefore shows that even $1+1$ dimensional Bethe ansatz solvable models may be difficult to computationally simulate classically, at least in the presence of intermediate measurements. Therefore integrable models (even those which are Bethe ansatz solvable) are not necessarily computationally simple.

Note that Bremner-Jozsa-Shepherd also achieve computational hardness with a unitary space described by polynomially many parameters [14]; however their model is incomparable to a $1+1$ dimensional quantum field theory so is not Bethe ansatz solvable, nor do their gates satisfy the Yang-Baxter equation. Also note that in general there are solutions to the Yang-Baxter equation that are known to be BQP universal, e.g., topological quantum computing in $2+1$ dimensions [21]. However these are not Bethe ansatz solvable. Also, prior work has shown that all unitary solutions to the Yang-Baxter equation on qubits and some solutions on qudits [7] are efficiently classically simulable. These works $[7,21]$ are incomparable to our results as they consider the parameter independent Yang-Baxter equation, whereas our model obeys the "parameter dependent" Yang-Baxter equation (i.e. values of $c$ and $s$ allowed depend on the momenta of the particles). Furthermore the systems considered in this paper and in [21] are not defined over tensor products of qudits, while [7] consider solutions over tensor products of qudits.

Finally, we consider classical versions of this model, where a base $\mathrm{AC}^{0}$ machine can query a deterministic, probabilistic, or nondeterministic ball-permuting oracle. Inputs to a deterministic ball permuting oracle are lists of swaps, and outputs are the permutations that are resulted from the application of swaps in order.

\footnotetext{
${ }^{5}[17]$ provide a nice introduction to this subject.

${ }^{6}$ For example, in classical mechanics total energy and total momentum are conserved quantities.

${ }^{7}$ In our model unitary evolution is the same as the unitary scattering matrix which relates input amplitudes to output amplitudes.
}

A randomized ball permuting oracle also takes a list of probabilities as input, applies the swaps probabilistically and outputs the final permutation. The model corresponding to the deterministic ball permutation is proved to be equivalent to $L$ (log-space) Turing machines. The randomized ball permutation model, on the other hand, can simulate BPL (Bounded-error probabilistic log-space) machines efficiently. We also show that a machine from the class Almost $L$ ( $L$ relative to a random oracle) can efficiently simulate randomized ball permutation. So the power the randomized ball permuting model lies between BPL and AlmostL. We raise the open problem of whether this can be simulated in $\mathrm{P}$. We also consider a nondeterministic version of the ball-permuting model. We find this to be equivalent to NP, unless the swaps are between adjacent balls only, in which case it can be simulated in $\mathrm{P}$ using nontrivial planar graph algorithms.

\section{MODELS AND MOTIVATIONS}

In this section we define the quantum ball-permuting model as a model of quantum computation, and then briefly explain how it models actual physical processes. The basic operations of this model are quantum swaps, as we will define them shortly. To obtain a point of comparison with the classical world we analyze the power of deterministic, randomized and non-deterministic swaps separately in section 4 .

The computational basis states in the ball permuting model are all $n$ ! possible permutations on an $n$-element set (1.e., $S_{n}$ ). We start out in the state

$$
|1,2, \ldots, n\rangle \text {. }
$$

At each time step, the rule is: we get to pick any adjacent ${ }^{8}$ pair of the $n$ registers in our quantum state, and then apply an $\left(n^{2}-\right.$ $n) \times\left(n^{2}-n\right)$ unitary transformation that, for every pair of distinct labels $x \neq y$, maps

$$
|x, y\rangle \rightarrow c|x, y\rangle+i s|y, x\rangle
$$

where $c$ and $s$ are any two real numbers ${ }^{9}$ satisfying $c^{2}+s^{2}=1$. (We get to pick whichever $c$ and $s$ we like for each gate operation. However, $c$ and $s$ can't vary depending on the labels $x$ and $y$. If one does allow for $c$ and $s$ to depend on $x$ and $y$, then in the appendix of the full version of our paper [3] we show one recovers BQP).

Finally, we measure all $n$ registers in the computational basis, and feed them to a classical computer for postprocessing.

We can represent the computational basis by the kets $|\sigma\rangle$ for any permutation $\sigma \in S_{n}$. We denote this Hilbert space by $\mathbb{C} S_{n}$. A quantum swap between $t$ and $t+1$ 'th registers depends on one real parameter $\theta$ and is represented by the operator

$$
X(\theta, t)=\cos \theta I+i \sin \theta L_{(t, t+1)} .
$$

Here $I$ is the identity operator, and $L_{\sigma}$ is a representation of $\mathbb{C} S_{n}$ with the action ${ }^{10}$

\footnotetext{
${ }^{8}$ We get the same model if we allow swaps between any pairs. This is because we can simulate general swaps with adjacent ones.

${ }^{9}$ Note that up to a global phase this is the most general form of amplitudes we can consider which results in a unitary partial swap gate.

${ }^{10}$ The operator $L$ acts from left to right on the basis of the Hilbert space. We can also talk about right multiplications $R(\tau)$ which map $|\sigma\rangle \mapsto\left|\sigma \circ \tau^{-1}\right\rangle$ for permutations $\sigma$ and $\tau$. The importance of these operators become clear in Section 3.2.2
} 


$$
L_{\sigma}|\tau\rangle=|\sigma \circ \tau\rangle
$$

The idea of this model is to capture $n$ distinguishable particles moving around on a line (1.e., in $1+1$ dimensions). The only state that we care about is the order of the particles in the line. The $x$ 'th register of the quantum computer stores the label of the $x$ 'th particle, if the particles are listed in order from left to right. Whenever two particles meet, one of two things can happen: the particles can reflect, or they can pass through each other. The first happens with amplitude $c$, while the second happens with amplitude $i s$.

We also consider a restricted version of this model, in which each particle $1 \ldots n$ has its own velocity $v_{1} \ldots v_{n}$, and particles can only interact if they collide. For instance if particle 2 is initially moving to the right, and all other particles are stationary, then particle 2 will only interact with particles to its right, and cannot interact with particles to its left. This model captures the power of scattering experiments with $\mathrm{n}$ distinguishable particles and repulsive (hardcore) interactions. This is illustrated in Figure 1. Many physical systems in both condensed matter theory $[13,43]$ and quantum field theory [44, 45] have similar behavior as this restricted model, as they describe the physics of particles scattering on a line with short-range interactions. Moreover, our proof of computational hardness for this model also works for scattering qutrits on a line, if one uses the "stationary programming" technique discussed in the appendix of the full version of our paper [3]. We leave open the question of whether there is a viable experimental realizaton of this model.

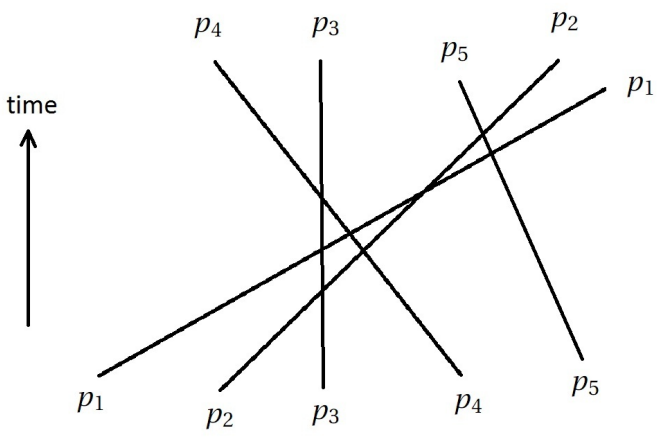

Figure 1: Five particles with momenta $p_{1}, p_{2}, \ldots, p_{5}$ moving on a line. At each intersection between these lines there is an interaction modeled by a ball-permuting gate. No other interactions are allowed.

Furthermore, as described in the appendix of the full version of our paper [3], if one rigorously defines this model, one finds the parameter $\theta$ in the exchange interactions cannot be arbitrary. Suppose we define a new parameter $z$, called the rapidity, which is the relative velocity of the colliding particles. Then we find that the exchange interaction must have $\theta=\tan ^{-1}(z)$. Furthermore, if we let $R(z, t)=X\left(\tan ^{-1}(z), t\right)$, then we additionally find our quantum operations must satisfy

$$
R\left(z_{1}, 1\right) R\left(z_{2}, 2\right) R\left(z_{3}, 1\right)=R\left(z_{3}^{\prime}, 2\right) R\left(z_{2}^{\prime}, 1\right) R\left(z_{1}^{\prime}, 2\right) .
$$

This is known as the parameter-dependent Yang-Baxter equation. We can confirm that the only nontrivial solution to this equation is, $z_{1}=z_{1}^{\prime}, z_{3}=z_{3}^{\prime}$, and $z_{2}=z_{2}^{\prime}=z_{1}+z_{3}$. Therefore this equation constrains the values of $z$ (and therefore the values of $\theta$ ) allowed in interactions in the model.

As a result, we will consider two types of models in this paper: one in which we imagine we can set $\theta$ to any value we choose for each interaction, and another based on the physics of colliding particles in which the interactions are constrained by both their velocities and the Yang-Baxter equation. The former model is much simpler to define and work with, while the latter model is more directly related to the physics of interacting particles on a line.

\section{THE QUANTUM BALL PERMUTING MODEL}

Based on the model introduced in Section 2, we will now formally define quantum ball-permuting complexity classes. We analyze their power with standard and arbitrary initial states in Sections 3.1 and 3.2, respectively. These sections assume that one can set the amplitudes $c$ and $s$ to arbitrary values for each interaction. Then, in Section 3.3, we analyze the complexity of this model when the interactions are constrained to obey the velocity constraints and the Yang-Baxter equations introduced at the end of Section 2.

Definition 3.1. QBall is the class of languages $L \subseteq\{0,1\}^{\star}$ for which there exist polynomial time Turing machines $M$ and $N$ such that on any input $x \in\{0,1\}^{\star}, M$ outputs the description of a ball permuting quantum circuit $C$ consisting of $\operatorname{poly}(n)$ partial swap gates such that, if $N$ is run on inputs consisting of $\operatorname{poly}(n)$ samples from $C$ in the computational basis

- If $x \in L$ then $N$ accepts with probability at least $\frac{1}{2}+\frac{1}{\operatorname{poly}(n)}$

- Otherwise if $x \notin L$ then $N$ accepts with probability at most $\frac{1}{2}-\frac{1}{\operatorname{poly}(n)}$.

Likewise, RQBall is defined to be the subclass of QBall where the gates of the ball permuting circuits are constrained to satisfy the Yang-Baxter equation.

The exact power of the classes QBall and RQBall each may depend on the input states allowed. In section 3.1 we analyze the power of the model when it start from a basis state and in section 3.2 we analyze it for arbitrary initial states.

\subsection{Standard Initial States}

In this section we will consider the power of QBall when the initial state is the identity permutation $|12 \ldots n\rangle$. We observe the following containments for the ball permuting complexity classes

Theorem 3.2. RQBall $\subseteq \mathrm{QBall} \subseteq \mathrm{BQP}$.

The containment RQBall $\subseteq \mathrm{QBall}$ is trivial. For $\mathrm{QBall} \subseteq \mathrm{BQP}$ note that we can represent labels with binary strings using $O(\log n)$ qubits. By the Solovay-Kitaev theorem [16], any unitary on $O(\log n)$ qubits can be implemented using a polynomial-size quantum circuit, and therefore a BQP circuit can simulate a QBall circuit. Therefore the power of QBall is upper bounded by BQP; this model is no more powerful than standard quantum computing. 
We will now show that starting from the initial state $|1,2, \ldots, n\rangle$ the power of QBall is likely much weaker than that of BQP, because one can efficiently estimate individual amplitudes in QBall to $\frac{1}{\text { poly }}$ error in the complexity class DQC1, i.e. the class of quantum computations that can be performed with one clean qubit and $n-1$ maximally mixed qubits. DQC1 is believed to be a substantially weaker complexity class than BQP. (For a discussion of DQC1 see the appendix of the full version of our paper [3].) In contrast, for circuits over qubits it is BQP-hard to compute individual amplitudes to $\frac{1}{\text { poly }}$ accuracy. The following observation is crucial for the establishment of our result relating QBall and DQC1:

LEMMA 3.3. If $C$ is any composition of $X$ ball permuting operators over $\mathbb{C S}_{n}$, then $C|123 \ldots n\rangle=|123 \ldots n\rangle$ if and only if $C=I$.

Proof. Suppose that $C|123 \ldots n\rangle=|123 \ldots n\rangle$, then permuting the labels arbitrarily gives $C|\sigma\rangle=|\sigma\rangle$ for all permutations $\sigma \in$ $S_{n}$.

The main result is the following:

THEOREM 3.4. There is a DQC1 algorithm which takes as input a description of a ball permuting circuit $C$ over $\mathbb{C} S_{n}$, and which outputs a complex number $\alpha$ such that $|\alpha-\langle 123 \ldots n|C| 123 \ldots n\rangle| \leq \frac{1}{\operatorname{poly}(n)}$, with high probability ${ }^{11}$.

Proof. (Sketch) For any ball-permuting circuit, we know that for any two permutations $\pi$ and $\pi^{\prime},\langle\pi|C| \pi\rangle=\left\langle\pi^{\prime}|C| \pi^{\prime}\right\rangle$. This implies that $\langle 123 \ldots n|C| 123 \ldots n\rangle=\frac{\operatorname{Tr}(C)}{n !}$. On the other hand, Knill and Laflamme [29] showed for any quantum circuit $U$ on $n$ qubits composed of polynomially many gates, a DQC 1 circuit can output a bit which is 1 with probability $\frac{1}{2}+\left|\frac{\operatorname{Tr}(U)}{2^{n+1}}\right|$, and therefore estimate $\left|\operatorname{Tr}(U) / 2^{n}\right|$ to $1 /$ poly additive error with postprocessing. Therefore it suffices to create a qubit unitary $U$ on $n$ qubits with the same trace as $C$ on $n$ particles, where $2^{n+1}$ is approximately the same as $n$ !. Fortunately it is possible to do this using a carefully chosen encoding of permutations with qubits. In particular, we use an encoding of permutations that is both compressed and local. By compressed we mean that it uses $\log (n ! \operatorname{poly}(n))$ bits, and by local we mean that we can use polynomial-size quantum circuits to simulate each quantum swap. We discuss this in detail in the appendix of the full version of our paper [3].

A similar argument holds for arbitrary amplitudes $\left\langle\sigma|C| \sigma^{\prime}\right\rangle$. This is followed by the reduction $C \mapsto L_{\sigma} C L_{\sigma^{\prime-1}}$ to the above problem. Therefore DQC1 can efficiently estimate amplitudes in the QBall model to $1 /$ poly accuracy. In contrast, for qubits, computing individual amplitudes to 1 /poly accuracy is BQP-hard. Therefore this is evidence that QBall is a weaker model of computation than BQP.

Note however Theorem 3.4 does not imply QBall $\subseteq$ DQC1 as decision languages, because we have only shown how to compute individual amplitudes of QBall in DQC1, while the decision class QBall may accept or reject based on an exponential sum of amplitudes. Likewise, Theorem 3.4 does not immediately imply a DQC1 machine could sample from the output distribution of a

\footnotetext{
${ }^{11}$ Furthermore, the DQC1 algorithm is able to find additive approximations for both the real and and imaginary values of the amplitude, separately.
}

QBall circuit. Whether it is possible to efficiently sample the output distributions of ball permuting circuits with DQC1 computation is unknown.

We conjecture that this result can be generalized to a wide variety of quantum models based on group algebras. More precisely, consider a group $G$, with identity element $e$. Then construct the Hilbert space $\mathcal{H}_{G}$ with orthonormal basis $\{|g\rangle: g \in G\}$. Let $\mathbb{C} G$ be the (left) group algebra, and $x \in \mathbb{C} G$. Then $\langle e|x| e\rangle=\frac{1}{|G|} \operatorname{Tr}(x)$, which is a reduction to the computation of normalized trace. If one has a compressed and local encoding of $G$ as described in the proof of Theorem 3.4, then this would imply that amplitudes of computations over $\mathbb{C} G$ can be simulated in DQC1 as well. We leave this is an open problem.

\subsection{Arbitrary Initial States}

We already observed that the model we obtain seems to be restricted if one starts with and measures according to the computational basis. In this section, we further examine how the power of ballpermuting model depends on the input states. We first give a simple construction showing that QBall is universal for BQP when given particular input states. The proof is based on DiVincenzo et al.'s result that the exchange interaction is universal for quantum computing [18]. We then provide a partial classification of the power of QBall on different input states using the representation theory of $S_{n}$. This requires substantial work in representation theory, and is the main technical contribution of the paper. In Section 3.2.2 we describe a number of input states which boost the power of QBall up to BQP. We then describe some other input states which yield a model intermediate between DQC1 and BQP in Section 3.2.3.

3.2.1 A simple proof that $\mathrm{QBall}=\mathrm{BQP}$ on arbitrary initial states. First of all, building on DiVincenzo et al.'s result that the exchange interaction is universal for quantum computing [18], we observe that when the initial state need not be a computational basis state, the quantum ball permuting model has the full power of BQP. The proof uses the notion of encoded universality: although our model does not allow arbitrary unitaries on the Hilbert space $\mathbb{C} S_{n}$, it can simulate arbitrary unitaries on certain subspaces of $\mathbb{C} S_{n}$ which encode qubits. We can therefore perform universal quantum computation on the encoded subspace, assuming our inputs are allowed to lie in the encoded subspace. This can be summarized by the following theorem:

THeOrem 3.5. If $\mathrm{QBall}$ is allowed to have non-basis input states, then $\mathrm{QBall}=\mathrm{BQP}$.

The authors of $[10,18]$ showed that one could use the exchange interaction to simulate one logical qubit using three physical qubits by the following encoding

$$
\left|0_{L}\right\rangle:=\frac{|010\rangle-|100\rangle}{\sqrt{2}}
$$

and,

$$
\left|1_{L}\right\rangle:=\frac{|010\rangle+|100\rangle-2|001\rangle}{\sqrt{6}} .
$$

Moreover the author of [18] showed how to implement an approximate CNOT on logical qubits using exchange interactions. This 
result was further improved in [19], where the authors found closed form expressions for this implementation.

In our model, we mimic this encoded universality construction for qubits using permutations. Specifically, to encode a logical qubit, we use permutations of a three-element set. We let the permutation labels 1 and 2 represent the qubit state $|0\rangle$ and the permutation label 3 represent the qubit state $|1\rangle$. We then symmetrize over the labels which represent with zeros and over the labels which represent with ones, to obtain states over $\mathbb{C} S_{3}$ which represents each basis state $|001\rangle,|010\rangle,|100\rangle$ used in DiVincenzo et al.'s construction. For example we represent the qubit state $|001\rangle$ with the state $|123\rangle+|213\rangle$, and we represent the state $|010\rangle$ with the state $|132\rangle+|231\rangle$. To encode logical zero and logical 1, we use DiVincenzo et al.'s encoding, ported over to permutations using the above correspondence. One can check this simulation works because the only operators being used in both models are permutations. For $n>3$ qubits we use the same symmetrization idea to simulate exchange interactions on $\left(\mathbb{C}^{2}\right)^{\otimes n}$; a detailed proof of this fact in given in the appendix of the full version of our paper [3].

Note that an alternative construction for encoded universality can be obtained using the path representations given in the appendix of the full version of our paper [3], which stands in line with the representation theory discussed in the next section. However, DiVincenzo et al.'s construction has the advantage of being more explicit. More specifically, we can encode $\left|0_{L}\right\rangle$ with the path $|u d u d\rangle$ and $\left|1_{L}\right\rangle$ with $|u u d d\rangle$. Here $u$ is corresponds to one step up, and $d$ is one step down. Using the result of next Section we know that we can apply $S U(V)$ on the space of these paths. Therefore to simulate BQP first initialize in $|u d u d \ldots u d u d\rangle$, implement sequences of gates to mimic arbitrary rotation and entangling two qubit gates on the encoded qubits and sample a path from the output. Note that the construction of these gates directly follow from theorem 3.6 of next Section. This encoding of qubits is also very similar to Aharonov and Arad's [4] construction for proving BQP-completeness for the evaluation of Jones polynomial.

3.2.2 Partial classification of input states which make QBall = BQP. Our particular contribution in this section is to demonstrate a partial classification for the computational power of this model according to different initial states. Our objective is to demonstrate that different input states lead to different interesting models of computation. This classification is obtained using the representation theory of the symmetric group. (For a brief review of this theory see the appendix of the full version of our paper [3]).

A representation of a group $G$ is a homomorphism : $G \rightarrow G L(V)$, for some vector space $V$, which obeys the same multiplication rule as the group law. We interchangeably refer to $V$ or the homomorphism itself as the representation. The regular representation of $S_{n}$ with left action is according to the homomorphism $L: S_{n} \rightarrow G L\left(\mathbb{C} S_{n}\right)$, with the map $L_{g}:|h\rangle \mapsto|g . h\rangle$. Similarly, the right action $R: S_{n} \rightarrow G L\left(\mathbb{C} S_{n}\right)$, is according to the map $R_{g}:|h\rangle \mapsto\left|h \cdot g^{-1}\right\rangle$. An invariant subspace is a subspace that is stable under the action of a particular representation, 1.e., the image of this subspace under the action of the group is equal to the subspace itself. A representation is called irreducible if its only invariant subspaces are the singleton $\{0\}$ and the representation itself.
Under these (left and right) regular representations the Hilbert space $\mathbb{C} S_{n}$ decomposes into irreducible representations as

$$
\mathbb{C} S_{n} \cong \bigoplus_{\lambda+n} V_{\lambda} \otimes X_{\lambda}
$$

where each $\lambda$ is a partition of the number $n$ : that is, a list of nonnegative integers in non-ascending order summing to $n$. $\lambda \vdash n$ means that $\lambda$ is a partition of $n . V_{\lambda} \otimes X_{\lambda}$ is a summand of the decomposition the tensor product of two vector spaces with $\operatorname{dim}\left(V_{\lambda}\right)=$ $\operatorname{dim}\left(X_{\lambda}\right)=: d_{\lambda}$ and $\sum_{\lambda} d_{\lambda}^{2}=n$ !.. Interestingly, the left actions of $S_{n}$ only acts on $V_{\lambda}$ 's and act trivially on the $X_{\lambda}$ 's. $X_{\lambda}$ 's are called the multiplicity spaces corresponding to each $V_{\lambda}$, as they enumerate all the irreducible representations isomorphic to $V_{\lambda}$.

Denote the Lie group generated by ball-permuting operators with $G_{n}$, and the projection of $G_{n}$ onto $V_{\lambda}$ with $G_{n}\left(V_{\lambda}\right)$. Note that $G_{n}\left(V_{\lambda}\right)$ is unitary, as it corresponds to the action of $G_{n}$ on the subspace of the irrep $V_{\lambda}$.

THEOREM 3.6. If $\lambda \vdash n$ or its transposed partition consists of two parts, then $S U\left(V_{\lambda}\right) \subseteq G_{n}\left(V_{\lambda}\right)$.

Proof. (Sketch) By induction, we first prove the statement for $n=3$. We then use subgroup adapted Young-Yamanouchi basis which manifests the branching rule (discussed in the appendix of the full version of our paper [3]), along with decoupling lemma and bridge lemma of Aharonov and Arad [4] to deduce the statement of this theorem for each subspace one-by-one. This procedure fails for subspaces corresponding to general partitions with more than two rows (columns).

The transpose of a partition $\lambda$ is a partition whose rows are the columns of $\lambda$ and whose columns are the rows of $\lambda$.

Therefore, if the input state to QBall corresponds to an irreducible representation which a) consists of two parts and b) is sufficiently large in dimension, then by Theorem 3.6 one can perform encoded universal computation on this input state. The description of these input states is given in the appendix of the full version of our paper [3]. We believe that the result can be extended to partitions with more than two rows or columns, however, the tools we used are restricted and we need more ideas to achieve this goal. We leave this result as an open question.

3.2.3 Some new intermediate quantum computing models. We saw in Section 3.1 that for a ball permuting circuit $C$, the amplitude $\langle 123 \ldots n|C| 123 \ldots n\rangle$ can be additively approximated using a DQC1 algorithm. Moreover, last Section asserted that for specific partitions $\lambda$, if $|\psi\rangle$ is a separable state over the partition $V_{\lambda} \otimes X_{\lambda}$, then it is BQP-complete to read the amplitude $\langle\psi|C| \psi\rangle$ within additive error. In this Section we provide evidence that the ball-permuting model along specific subspaces of $\mathbb{C} S_{n}$ yields a model of computing that is intermediate between DQC1 and BQP.

Suppose that instead of the computational basis, we initialize the ball-permuting model with the projection of the state $|123 \ldots n\rangle$ onto an irrep $\lambda$. Denote this (normalized) state by $|\lambda\rangle$. Then we apply a sequence of ball-permuting gates, and at the end of the computation we sample a pair of tableaux in $V_{\lambda} \otimes X_{\lambda}$ according to the Young-Yamanouchi basis (see the appendix of the full version of our paper [3] for a review). Inspired by this model, we formally define the following complexity class: 
Definition 3.7. SampQBall $(\lambda)$ is the class of problems that are solvable in polynomial time using polynomially many samples from the above model.

The exact power of this model is unknown, however, we motivate reductions to an interesting complexity class that is intermediate between DQC1 and BQP. We can also define the following computational problem:

Definition 3.8. (QBall $(\lambda))$ given a ball permuting circuit $C$, and a partition $\lambda$, evaluate an additive approximation to $\langle\lambda|C| \lambda\rangle$.

Interestingly, the initial state $|123 \ldots n\rangle$ is equally supported on all of the irreps $\lambda$ of the decomposition $\mathbb{C S}_{n} \cong \bigoplus_{\lambda \vdash n} V_{\lambda} \otimes X_{\lambda}$, and moreover it is maximally entangled over each partition $V_{\lambda} \otimes$ $X_{\lambda}$. Also, notice that (as mentioned in the last Section) all ballpermuting operators only act on $V_{\lambda}$ parts, and act trivially on $X_{\lambda}$ 's, the multiplicity spaces ${ }^{12}$.

In short, we find that the model operates on one half of a maximally entangled state, while leaving the other half untouched. At the end of the computation, one measures both halves of the maximally entangled state. To investigate the power of this model, as a toy model, we consider a restricted model of quantum computing. Imagine we have access to only one half of the state

$$
|\psi\rangle=\frac{1}{2^{n / 2}} \sum_{x \in\{0,1\}^{n}}|x\rangle|x\rangle .
$$

Suppose we can perform standard unitary quantum computation on the left half of the state, but one cannot access the right half of the state. At the end of the computation, then we get to measure both halves of the state in the computational basis.

This model seems very similar to the class DQC1, because if $C$ is a quantum circuit on the $n$ active qubits, then

$$
\langle\psi|C| \psi\rangle=\frac{\operatorname{Tr}(C)}{2^{n}},
$$

the normalized trace. This is simply because the reduced density matrix of the left half of the state is maximally mixed. We have seen in Section 3.1 that evaluation of such a trace within additive error is complete for the class DQC1. Therefore if we define the trace computing class:

Definition 3.9. (Trace computing quantum polynomial time) TQP is the class of problems that are polynomial-time reducible to additive approximation of $\langle\psi|C| \psi\rangle$, the normalized trace of the matrix. Also SampTQP is the class of problems that are solvable with high probability using polynomially many samples from $C|\psi\rangle$, in the computational basis.

Then we trivially find TQP = DQC1. However, the class SampTQP seems to be more powerful than DQC1. The reason is that one gets to measure the right half of the state at the end of the computation; this is not an ability one has in DQC1. To put it another way, DQC1 is defined using maximally mixed states as inputs, which is equivalent to performing your computation on a random basis

\footnotetext{
${ }^{12}$ Intuitively, the situation resembles a quantum/classical hybrid memory in the sense of [31], where classical bits enumerate the name $\lambda$ corresponding to the particular irrep, and the quantum memory corresponds to a bipartite system-environment Hilbert space $V_{\lambda} \otimes X_{\lambda} ; V_{\lambda}$ plays the role of a system, and $X_{\lambda}$ is its environment which is inert and also maximally entangled with the system.
}

state $|x\rangle$. SampTQP is equivalent to performing your computation on a random $|x\rangle$, but at the end of the computation, you get to learn which $|x\rangle$ you started with. As a result, it appears that SampTQP is intermediate between DQC1 and BQP. Note that SampTQP is BQP-universal under postselection as well.

We first observe that:

THEOREM 3.10. If $\lambda$ is a partition with two equally sized rows, then $\mathrm{QBall}(\lambda) \in \mathrm{TQP}$.

Proof. (Sketch) following the proof of theorem 3.4 we use a compressed and local representation of standard tableaux with two rows of length $n / 2$ using strings of bits. Specifically, suppose we represent standard tableaux on two equal-sized rows by bit strings of length $n$. The $i$ th entry represents whether the number $i$ appears on the top or bottom row. There are $2^{n}$ such strings and $2^{n} / \operatorname{poly}(n)$ valid tableaux, so although many of the strings do not represent valid tableaux, a $1 /$ poly fraction do represent valid tableaux. So this encoding is very efficient. Furthermore, it is local in the sense that to exchange two labels, one simply exchanges their corresponding bits. Finally, it is easy to test if a string is a valid encoding. Additionally, it one can compute using $O(\log n)$ ancillas the axial distance (as defined in the appendix of the full version of our paper [3]) between two labels in the tableau. Following the techniques of the full version of our paper [3], the existence of such an encoding implies membership in DQC1, as one can simulate the action of ball-permuting gates on these basis, and use a DQC1 procedure to evaluate an additive approximation to $\langle\lambda|C| \lambda\rangle$. See the appendix of the full version of our paper [3] for details.

We conjecture that the power of QBall on all such input states $|\lambda\rangle$ is contained in TQP. However, it is difficult to prove this fact because DQC1 is defined using qubits, while the $|\lambda\rangle$ basis is labeled by the Young-Yamanouchi basis (See the appendix of the full version of our paper [3]). As in Section 3.1, in order to prove QBall is in TQP on this input states, we would need to find an encoding of the Young-Yamanouchi basis which is both extremely compressed and local for arbitrary Young diagrams. We mention this is an open problem in the appendix of the full version of our paper [3].

Next, we build a connection between the classes SampQBall $(\lambda)$ and SampTQP:

THEOREM 3.11. If $\lambda$ is a partition with two equally sized rows, $\operatorname{SampQBall}(\lambda) \subseteq$ SampTQP.

Proof. (Sketch) as in the proof of theorem 3.10, we use a compressed and local representation of tableaux with two equally sized rows using binary strings to simulate the action of ball permuting gates on $V_{\lambda}$ in a succinct space, and sample from the output distribution in the computational basis. After postprocessing, with high probability the sampled string corresponds to a valid sample from SampQBall $(\lambda)$.

SampTQP is a restricted model of computation on qubits, and is an interesting model on its own right. We can immediately observe the following 


\section{Theorem 3.12. DQC $1 \subseteq \mathrm{SampTQP} \subseteq \mathrm{BQP}$.}

Proof. SampTQP $\subseteq \mathrm{BQP}$ is immediate. To see why DQC1 $\subseteq$ SampTQP, assume optimistically that the first active bit is in the pure state $|0\rangle$. Then, at the end, when we measure we will find out whether or not the assumption was correct, and it will have been with probability $1 / 2-$ in which case we will have performed the same computation as $\mathrm{DQC}_{1}$. Hence if the $\mathrm{DQC}_{1}$ machine decides a language with bias $1 /$ poly, then this TQP simulation decides the language with bias 1 /poly as well.

\subsection{The Power of Yang-Baxter Ball Permutations}

In this section, we consider the complexity class RQBall, in which the partial swap interactions are constrained by the physics of distinguishable particles. In particular, as discussed briefly in Section 2 and in detail in the appendix of the full version of our paper [3], the amplitudes $c$ and $s$ are constrained by the relative velocities of the interacting particles, and only those particles which collide can interact. This physically corresponds to a two-dimensional integrable quantum field theory of $\mathrm{n}$ distinguishable particles on a line.

Before presenting these results, we first note that we do not expect this model to be capable of universal quantum computing, because the dimensionality of the space of unitary operations allowed in this model is too small (this Theorem is proved in the appendix of the full version of our paper [3] in more detail):

THEOREM 3.13. Let $Q_{n}$ be the Lie group generated by planar YangBaxter quantum circuits ${ }^{13}$ over $n$ labels, then $Q_{n}$ as a manifold is isomorphic to the union of $n !$ manifolds, each with dimension at most $n$.

Proof. (Sketch) This follows by noticing that if the operators satisfy the Yang-Baxter equation, then the final unitary operator only depends on $n$ real parameters, namely the initial momenta of the particles.

In other words, if the interactions of the ball-permuting model satisfy the Yang-Baxter equation, then the group of unitary operators generated on $n$ labels corresponds to a Lie group of linear dimension. In contrast the standard definition of BQP allows unitaries of dimension exponential in the number of qubits. This makes it unlikely that one could do universal quantum computation in this model; however, it does not imply efficient classical simulation.

To the contrary, in this section we establish some hardness results for classical simulation of the class RQBall following the strategy of Aaronson and Arkhipov [2], Bremner-Jozsa-Shepherd [14] and others. In particular, we show that a classical computer cannot sample from the same distribution as these circuits unless the polynomial hierarchy collapses to the third level. Our argument makes use of two technical concepts. The first is intermediate demolition measurements. A demolition measurement is a measurement of the label at a particular location, where after the label is measured, it is removed from the computation ("demolished"), i.e. the Hilbert space becomes $\mathbb{C} S_{n-1}$ rather than $\mathbb{C} S_{n}$. The second is the concept of postselection (discussed in greater detail in the appendix of the full

\footnotetext{
${ }^{13}$ For a precise definition see the full version of our paper [3].
}

version of our paper [3]). Here postselection is the (non-physical) ability to specify the outcome of a measurement in advance. We show that postselected RQBall circuits with intermediate demolition measurements are capable of universal postselected quantum computation. By the techniques of $[2,14]$, this immediately implies that a classical computer cannot efficiently sample from the same probability distribution unless the polynomial hierarchy collapses. The basic reason is that postselected quantum computation (denoted by the complexity class PostBQP) is more powerful that postselected classical computation (denoted by the class PostBPP) assuming PH does not collapse. Therefore if a classical computer could sample from the same distribution, then by postselecting the simulation of the quantum device, that would solve a PostBQP problem in PostBPP, which causes $\mathrm{PH}$ to collapse.

More formally, we consider the following complexity class:

Definition 3.14. PostRQBall is the class of decision problems that are solvable in polynomial-time using postselected Yang-Baxter ball collision circuits with intermediate demolition measurements. In this model the input state is a special initial state described in the appendix of the full version of our paper [3].

More specifically PostRQBall is the set of languages $L$ such that there exists a postselected quantum RQBall circuit, and a poly-timecomputable set of permutations $P$, such that if $\sigma$ is the permutation measured at the end of the computation, and $C$ is the event all intermediate measurements yield their postselected values, then

$$
\begin{aligned}
& \text { 1) } \operatorname{Pr}[C]>0 . \\
& \text { 2) if } x \in L, \operatorname{Pr}[\sigma \in P \mid C] \geq 2 / 3 \text {. } \\
& \text { 3) if } x \notin L, \operatorname{Pr}[\sigma \in P \mid C] \leq 1 / 3 .
\end{aligned}
$$

Likewise define SampRQBall to be the class of sampling problems that are solvable by polynomial time uniform Yang-Baxter ball collision circuits on arbitrary initial states and intermediate demolition measurements.

The central observation is the following theorem, stating that although the ball scattering model might be strictly weaker than $\mathrm{BQP}$, the postselected version is equal to PostBQP, which is equal to PP [1].

Theorem 3.15. PostRQBall $=$ PostBQP $=$ PP.

Proof. (sketch). In the appendix of the full version of our paper [3] we show how to use intermediate demolition measurements in RQBall circuits to simulate standard QBall circuits, assuming one can postselect the outcomes of the intermediate demolition measurements. Since QBall $=\mathrm{BQP}$ this implies BQP $\subseteq$ PostRQBall in an encoded sense. By postselecting this encoded BQP simulation we can simulate arbitrary PostBQP computations in PostRQBall as well.

From this, using the techniques of $[2,14]$, we immediately have the following corollary:

COROLLARY 3.16. There is no polynomial time randomized algorithm to simulate SampRQBall to within multiplicative constant error, unless $\mathrm{PH}$ collapses to its third level.

Proof. (sketch). If a BPP machine could sample from the same distribution as a SampRQBall circuit, then by postselecting the simulation, one could simulate PostRQBall $=\mathrm{PP}$ in PostBPP, and 
hence PostBPP $=$ PP. But by $[2,14]$ this would imply the collapse of $\mathrm{PH}$, because then $\mathrm{PH} \subseteq \mathrm{P}^{\mathrm{PP}}=\mathrm{P}^{\mathrm{PostBPP}} \subseteq \Delta_{3}$. Here the first containment is Toda's theorem [40] and the third follows from the fact PostBPP $\subseteq \Sigma_{\mathrm{P}}^{3}[8]$.

Therefore, despite the fact that the dimensionality of the space of unitaries in RQBall is small, RQBall computations cannot be efficiently simulated classically (up to multiplicative error) unless the polynomial hierarchy. Hence the RQBall model seems to be intermediate in power between BPP and BQP.

\section{COMPUTATIONAL COMPLEXITY OF THE CLASSICAL BALL PERMUTING MODEL}

To understand which features of the quantum ball-permuting model actually depend on quantum mechanics, in this section, we define a classical model of ball permuting and analyze its computational power. In this model input is a list of swaps in $S_{n}$, and the output is a permutation of $n$ labels. We consider three models - deterministic, randomized and nondeterministic for the application of swaps.

Before introducing our classical ball permuting models, we will first review some classical complexity theory. Recall that $L$ is the class of decision problems that are solvable on a log-space Turing machine. BPL is the class of decision problems that are solvable with bounded probability of error on a probabilistic logarithmicspace Turing machine, while AlmostL is defined as the class of languages that are decidable with bounded probability of error by a logarithmic-space Turing machine with access to a random oracle with probability 1 . Interestingly, AlmostL contains BPL, but the converse is not known. The issue is that an AlmostL machine can use the same specific polynomially-long random string over and over, which is not an ability that a BPL machine obviously has. Indeed, AlmostL is not even known to be contained in $P$, though it is contained in BPP.

A reversible Turing machine is a Turing machine such that for any of its computations, the nodes of its infinite configuration graph have in-degree and out-degree of at most 1 . Reversible log-space or RevL is the class of decision problems that are solvable by reversible log-space Turing machines. Lange, McKenzie, and Tapp [33] proved the following important result:

THEOREM 4.1. $\mathrm{L}=$ RevL.

We now define the classical ball-permuting model.

Definition 4.2.

- DBall is the class of languages that are decidable by an $\mathrm{AC}^{0}$ machine that can make a single query to a deterministic ball-permuting oracle”. Such an oracle takes as input a polynomially-long list of swaps $\left(i_{1}, j_{1}\right),\left(i_{2}, j_{2}\right), \ldots$ and returns the permutation obtained by applying those swaps in order to the initial permutation $(1,2, \ldots, n)$.

Also, DBall $a d j$ is the subclass of DBall where all swaps are adjacent elements only.

- RBall is the class of languages that are decidable, with bounded probability of error, by an $A C^{0}$ machine with a single query to a "randomized ball-permuting oracle". Such an oracle takes as input a polynomially-long list of swaps $\left(i_{1}, j_{1}\right),\left(i_{2}, j_{2}\right), \ldots$ along with a list of probabilities $p_{1}, p_{2}, \ldots$ It returns the permutation obtained by swapping $\left(i_{1}, j_{1}\right)$ with independent probability $p_{1}$, then swapping $\left(i_{2}, j_{2}\right)$ with independent probability $p_{2}$, and so on, starting from the initial permutation $(1,2, \ldots, n)$.

$\mathrm{RBall}_{a d j}$ is the subclass where the swaps are restricted to be adjacent ones only, and RBall ${ }_{a d j}^{\star}$ is the subclass where all probabilities are nonzero and also not equal to one.

We find that deterministic ball permuting captures L, while randomized ball permuting defines a class which is intermediate between BPL and AlmostL. We include sketches of these proofs, but details can be found in the appendix of the full version of our paper [3].

Theorem 4.3. DBall $=$ DBall $_{a d j}=\mathrm{L}=\operatorname{RevL}$

Proof. (Sketch) For DBall ${ }_{a d j}=$ DBall, just observe that we can easily implement any swap we want as a sequence of adjacent swaps. For $\mathrm{L} \subseteq$ DBall, we show how to simulate reversible logspace with swaps and then appeal to theorem 4.1. For DBall $\subseteq \mathrm{L}$, we use $L$ subroutines to keep track of each label one-by-one.

Theorem 4.4. $\mathrm{L} \subseteq \mathrm{BPL} \subseteq \mathrm{RBall}=\mathrm{RBall}_{a d j} \subseteq \mathrm{Almost} \mathrm{L} \subseteq \mathrm{BPP}$. However, if we let $\mathrm{RBall}(2)$ be the class where the $\mathrm{AC}^{0}$ machine is allowed to make two adaptive queries to the ball-permuting oracle, then $\mathrm{RBa}$ II $(2)=$ AlmostL.

Proof. (Sketch) RBall $=$ RBall $_{a d j}$ is proved similarly to the previous theorem. Also, for RBall $\subseteq$ AlmostL: we just run the $\mathrm{DBall} \subseteq \mathrm{L}$ simulation, except that any time we need to apply a probabilistic swap, we use the random oracle to decide whether to make it or not.

Finally, for $\mathrm{BPL} \subseteq$ RBall: we run the $\mathrm{L} \subseteq$ DBall simulation, except that whenever the $L$ machine wants a random bit in its memory, we use random swaps on every adjacent pair of elements $((1,2),(3,4), \ldots)$ to produce one.

Next, in order to understand the power of nondeterministic ballpermutation, we investigate the problem of deciding whether or not the output of an RBall computation has positive support on a specific permutation:

Definition 4.5. (Ball) Given a polynomially-long list of swaps $\left(i_{1}, j_{1}\right), \ldots,\left(i_{m}, j_{m}\right)$, and a list of indepedent probabilities $p_{1}, \ldots, p_{m}$ as inputs of a randomized ball-permuting oracle, decide if there is a positive probability that the oracle outputs a specific target permutation $\sigma \in S_{n}$. Ball $a d j$ is the restriction of the language when all the input swaps are adjacent ones, and Ball ${ }_{a d j}^{\star}$ is the language when we further restrict the probabilities to be strictly between 0 and 1.

First we prove that the problem is NP-complete for general swaps. We prove this by a reduction from "word problem for the product of permutations (WPPP)" which is known to be NP-complete. More precisely:

Definition 4.6. (WPPP) Given the set $\{1,2,3, \ldots, n\}=:[n]$, an ordered list of subsets $S_{1}, S_{2}, \ldots, S_{m} \subseteq[n]$ with $m=\operatorname{poly}(n)$, and a target permutation $\tau$ on $[n]$, the problem is to decide whether there exist permutations $\pi_{1}, \pi_{2}, \ldots, \pi_{m}$, with each $\pi_{j}$ acting on the labels 
of $S_{j}$ only and identity on the others, such that the combination $\pi_{1} \circ \pi_{2} \circ \ldots \circ \pi_{m}=\tau$.

Theorem 4.7. (Garey, fohnson, Miller, and Papadimitriou [22]) WPPP is NP-complete.

THEOREM 4.8. Ball ${ }_{a d j}$ and Ball are polynomial-time reducible to each other, and furthermore they are complete for the class NP.

Proof. (Sketch) To see polynomial-time equivalence between the two languages, we use adjacent swaps with probability 1 to simulate nonadjacent ones. To prove completeness for NP we use theorem 4.7 .

On the other hand, we find that the non-deterministic class Ball ${ }_{a d j}^{*}$ is contained in P. Our proof uses a reduction to planar case of the edge disjoint path problem.

Definition 4.9. (The edge disjoint path problem EDP) Given a directed graph $G$, with source and sink nodes $\left(s_{1}, t_{1}\right),\left(s_{2}, t_{2}\right), \ldots$, $\left(s_{m}, t_{m}\right)$, decide if there are paths from $s_{i}$ to $t_{i}$ for all $i \in[m]$, such that all the paths are edge-disjoint.

THEOREM 4.10. (Wagner and Weihe[41]) EDP for the case of planar graphs is decidable in linear time.

THEOREM 4.11. Ball ${ }_{a d j}^{\star} \in \mathrm{P}$

Proof. (Sketch) we use a linear time reduction to EDP. Given a list of swaps we construct a planar directed graph for which an edge disjoint path exists if and only if the target permutation is generated in the non-deterministic swaps.

In other words, given a list of swaps and a target permutation, it is in general NP-hard to decide if there is a way of constructing the target permutation out of the given swaps. If all the swaps are transpositions, the problem is decidable in linear time. Observe that our result about the language Ball gives an independent proof for:

COROLlary 4.12. The edge disjoint path problem in the non-planar case is NP-complete.

\section{DISCUSSION AND CONCLUSIONS}

We have explored several models of ball-permuting computation. We found the power of QBall depends on the input states allowed in the model, and we partially classified which input states allow QBall to perform universal quantum computation using the representation theory of $S_{n}$. Additionally, we found that even the highly restricted model of RQBall cannot be efficiently simulated classically unless PH collapses. This is surprising as the model RQBall admits unitary transformations of only polynomial dimension. Classically, we found that RBall is of power intermediate between BPL and AlmostL.

A natural open problem is to finish classifying the power of QBall on all input states. While we nearly completed this task, the tools we used for the classification are restricted in the sense that they can only take care of special subspaces and not the others. Another natural open problem is to determine if QBall with the starting state $|12 \ldots n\rangle$ is contained in DQC1 as a decision class. A list of further open problems can be found in the appendix of the full version of our paper [3].

\section{ACKNOWLEDGEMENTS}

We thank Robin Kothari for suggesting connections to the class DQC1 and Luke Schaeffer for helpful discussions regarding succinct encodings of permutations and tableaux. We thank an anonymous referee for helpful comments about integrable models. S.M. thanks Hossein Esfandiary for suggesting connections to factorial number system and Usman Naseer \& Hamed Pakatchi for useful comments on the physics.

\section{REFERENCES}

[1] Scott Aaronson. Quantum computing, postselection, and probabilistic polynomial-time. In Proceedings of the Royal Society of London A: Mathematical, Physical and Engineering Sciences 461, 3473-3482, 2005.

[2] Scott Aaronson and Alex Arkhipov. The computational complexity of linear optics. In Proceedings of the forty-third annual ACM symposium on Theory of computing (STOC '11), 333-342, ACM, 2011.

[3] Scott Aaronson, Adam Bouland, Greg Kuperberg and Saeed Mehraban. The computational complexity of ball permutations. arXiv:1610.06646, 2016.

[4] Dorit Aharonov and Itai Arad. The BQP-hardness of approximating the Jones polynomial. New Journal of Physics, 13(3):035019, 2011.

[5] Dorit Aharonov, Itai Arad, Elad Eban, and Zeph Landau. Polynomial quantum algorithms for additive approximations of the Potts model and other points of the Tutte plane. arXiv:quant-ph/0702008, 2007.

[6] Dorit Aharonov, Vaughan Jones, and Zeph Landau. A polynomial quantum algorithm for approximating the Jones polynomial. Algorithmica, 55(3):395-421, 2009.

[7] Gorjan Alagic, Aniruddha Bapat, and Stephen Jordan. Classical simulation of Yang-Baxter gates. 9th Conference on the Theory of Quantum Computation, Communication and Cryptography (TQC 2014), 161-175, 2014.

[8] Sanjeev Arora and Boaz Barak. Computational complexity: a modern approach. Cambridge University Press, 2009.

[9] D Bacon, J Kempe, DP DiVincenzo, DA Lidar, and KB Whaley. Encoded universality in physical implementations of a quantum computer. Proceedings of the International Conference on Experimental Implementation of Quantum Computation, Sydney, Australia (IOC 01), 257-264, Rinton Press, 2001.

[10] D Bacon, J Kempe, DA Lidar, and KB Whaley. Universal fault-tolerant computation on decoherence-free subspaces. Physical Review Letters, 85(8):1758-61, 2000 .

[11] Bela Bauer, Claire Levaillant, and Michael Freedman. Universality of single quantum gates. arXiv:1404.7822, 2014.

[12] Rodney J Baxter. Partition function of the eight-vertex lattice model. Annals of Physics, 70(1):193-228, 1972.

[13] Sougato Bose and Vladimir Korepin. Quantum gates between flying qubits via spin-independent scattering. arXiv:1106.2329, 2011.

[14] Michael J. Bremner, Richard Jozsa, and Dan J. Shepherd. Classical simulation of commuting quantum computations implies collapse of the polynomial hierarchy. Proceedings of the Royal Society of London A: Mathematical, Physical and Engineering Sciences, 467(2126):459-472, 2010.

[15] HJ Briegel, DE Browne, W Dür, R Raussendorf, and Maarten Van den Nest. Measurement-based quantum computation. Nature Physics, 5(1):19-26, 2009.

[16] Christopher M. Dawson and Michael A. Nielsen. The Solovay-Kitaev algorithm. Quantum Info. Comput., 6(1):81-95, January 2006.

[17] Marius de Leeuw and Constantin Candu. Lecture Notes for "Introduction to Integrability", 2013. Retrieved April 10, 2017 from ETH Zürich: http://edu.itp.phys.ethz.ch/fs13/int/

[18] David P DiVincenzo, Dave Bacon, Julia Kempe, Guido Burkard, and K Birgitta Whaley. Universal quantum computation with the exchange interaction. Nature, 408(6810):339-342, 2000.

[19] Bryan H Fong and Stephen M Wandzura. Universal quantum computation and leakage reduction in the 3-qubit decoherence free subsystem. Quantum Information \& Computation, 11(11-12):1003-1018, 2011.

[20] Lance Fortnow and John D. Rogers. Complexity limitations on quantum computation. Journal of Computer and System Sciences, 59(2):240-252, 1999.

[21] Michael Freedman, Alexei Kitaev, Michael Larsen, and Zhenghan Wang. Topological quantum computation. Bulletin of the American Mathematical Society, 40(1):31-38, 2003.

[22] Michael R Garey, David S Johnson, Gary L Miller, and Christos H Papadimitriou. The complexity of coloring circular arcs and chords. SIAM fournal on Algebraic Discrete Methods, 1(2):216-227, 1980.

[23] Daniel Gottesman, Alexei Kitaev, and John Preskill. Encoding a qubit in an oscillator. Physical Review A, 64(1):012310, 2001.

[24] Gordon James and Adalbert Kerber. The representation theory of the symmetric group. Reading, Mass, 1981 
[25] Stephen P Jordan. Permutational quantum computing. Quantum Information and Computation 10:470, 2010.

[26] Julia Kempe, Dave Bacon, Daniel A Lidar, and K Birgitta Whaley. Theory of decoherence-free fault-tolerant universal quantum computation. Physical Review A, 63(4):042307, 2001.

[27] Julia Kempe, David Bacon, David P DiVincenzo, and K Brigitta Whaley. En coded universality from a single physical interaction. Quantum Information \& Computation, 1(4):33-55, 2001.

[28] Julia Kempe and K Birgitta Whaley. Exact gate sequences for universal quantum computation using the XY interaction alone. Physical Review A, 65(5):052330 2002.

[29] Emanuel Knill and Raymond Laflamme. Power of one bit of quantum information. Physical Review Letters, 81(25):5672, 1998.

[30] Emanuel Knill, Raymond Laflamme, and Gerald J Milburn. A scheme for efficient quantum computation with linear optics. Nature, 409(6816):46-52, 2001.

[31] Greg Kuperberg. The capacity of hybrid quantum memory. IEEE Transactions on Information Theory, 49(6):1465-1473, 2003.

[32] Greg Kuperberg. Denseness and Zariski denseness of Jones braid representations. Geometry \& Topology, 15(1):11-39, 2011.

[33] Klaus-Jörn Lange, Pierre McKenzie, and Alain Tapp. Reversible space equals deterministic space. Journal of Computer and System Sciences, 60(2):354-367, 2000.

[34] Debbie W Leung. Quantum computation by measurements. International fournal of Quantum Information, 2(01):33-43, 2004.

[35] Tomoyuki Morimae, Keisuke Fujii, and Joseph F. Fitzsimons. Hardness of classically simulating the one-clean-qubit model. Physical Review Letters, 112:130502, Apr 2014.
[36] Michael E. Peskin and Daniel V. Schroeder. An Introduction to Quantum Field Theory. Perseus Books, 1995.

[37] Peter W Shor and Stephen P Jordan. Estimating Jones polynomials is a complete problem for one clean qubit. Quantum Information \& Computation, 8(8):681-714, 2008 .

[38] Matthias Staudacher. Review of ADS/CFT integrability, Chapter iii. 1: Bethe ansätze and the R-matrix formalism. Letters in Mathematical Physics, 99(1-3):191208, 2012.

[39] Barbara M Terhal and David P DiVincenzo. Adaptive quantum computation, constant depth quantum circuits and Arthur-Merlin games. Quantum Information \& Computation 4 (2): 134-145, 2004.

[40] Seinosuke Toda. PP is as hard as the polynomial-time hierarchy. SIAM fournal on Computing, 20(5):865-877, 1991

[41] Dorothea Wagner and Karsten Weihe. A linear-time algorithm for edge-disjoint paths in planar graphs. Combinatorica, 15(1):135-150, 1995.

[42] L-A Wu and DA Lidar. Power of anisotropic exchange interactions: Universality and efficient codes for quantum computing. Physical Review A, 65(4):042318, 2002.

[43] Chen-Ning Yang. Some exact results for the many-body problem in one dimension with repulsive delta-function interaction. Physical Review Letters, 19(23):1312, 1967.

[44] Zamolodchikov, Alexander B and Zamolodchikov, Alexey B. Relativistic factorized S-matrix in two dimensions having $\mathrm{O}(\mathrm{N})$ isotopic symmetry Annals of physics, 133(3): 253-291, 1979.

[45] Zamolodchikov, Alexander B and Zamolodchikov, Alexey B. Factorized Smatrices in two dimensions as the exact solutions of certain relativistic quantum field theory models. Nuclear Physics B, 120(2): 525-535, 1978. 